EGU21-13394

https://doi.org/10.5194/egusphere-egu21-13394

EGU General Assembly 2021

(c) Author(s) 2021. This work is distributed under

the Creative Commons Attribution 4.0 License.

\title{
Three-dimensional aseismic ruptures driven by fluid injection
}

\author{
Alexis Sáez ${ }^{1}$, Brice Lecampion ${ }^{1}$, Pathikrit Bhattacharya ${ }^{2}$, and Robert C. Viesca ${ }^{3}$ \\ 1'École Polytechnique Fédérale de Lausanne, Lausanne, Switzerland (alexis.saez@epfl.ch) \\ ${ }^{2}$ National Institute of Science Education and Research, Bhubaneswar, India \\ ${ }^{3}$ Tufts University, Medford, United States
}

\begin{abstract}
Injection-induced seismicity is usually observed as an enlarging cloud of seismic events that grows in a diffusive manner around the injection zone. These observations are commonly interpreted as the triggering of instabilities in pre-existing fractures and faults due to the direct effect of pore pressure increase (Shapiro, 2015), whereas poroelastic stressing is usually associated with the occurrence of seismic events beyond the plausible zone affected by pore pressure diffusion (Segall and Lu, 2015). However, an alternative triggering mechanism based on the elastic transfer of stress due to injection- induced aseismic slip has been recently proposed (Viesca, 2015; Guglielmi et al, 2015). Previous studies have shown that in critically stressed faults, the aseismic rupture front can outpace fluid diffusion (Garagash and Germanovich, 2012; Bhattacharya and Viesca, 2019), and in turn be the primary cause that controls the evolution of seismicity as it has been recently inferred from in-situ experiments of fluid injection (Duboeuf et al., 2017) and recent cases of injection-induced earthquakes (Eyre et al, 2019).
\end{abstract}

Despite the great relevance of aseismic slip on injection-induced seismicity, the conditions that control the three-dimensional propagation of aseismic ruptures are still poorly constrained. This is in part due to the challenge of solving such a 3D moving boundary problem in which both fault slip and rupture shape are unknown. Here, we study the mechanics of injection-induced aseismic ruptures on a planar fault characterized by a strength equal to the product of a constant friction coefficient and the effective normal stress. We systematically track the temporal evolution of the rupture area relative to the evolution of the pressurized zone and focus on the effect of the initial stress state and injection scenario. For injection at constant flux, we derive a semi-analytical solution for circular ruptures (for a Poisson's ratio equal to zero), which gives the ratio between the rupture radius and a nominal pore pressure front location, which we named as amplification factor $\lambda$. This amplification factor is a function of a unique dimensionless parameter that depends on the initial fault stress criticality and the fluid-induced overpressure. Then, we generalize the semi-analytical solution to the case of non-circular ruptures (for any value of the Poisson's ratio) by solving numerically for the spatiotemporal evolution of fault slip using a fully implicit boundaryelement-based solver with quadratic triangular elements. We show that the rupture front is nearly 
elliptical and the rupture area $A_{r}$ evolves in a self-similar diffusive manner such that $A_{r}(t)=4 \pi a \lambda^{2} t$, where $\alpha$ is the fault hydraulic diffusivity and $\lambda$ is the amplification factor for circular ruptures. The rupture area is greater than the nominal pressurized area if $\lambda>1$. The semi-analytical solution for the rupture area provides a unique opportunity for verifying numerical hydro-mechanical solvers. After, we investigate numerically the case of circular and non-circular ruptures driven by injection at constant pressure instead of constant flux. We show that the self-similar property of the rupture growth is lost under this injection scenario. 